Research Article

\title{
Study on Medication Rules of Traditional Chinese Medicine against Antineoplastic Drug-Induced Cardiotoxicity Based on Network Pharmacology and Data Mining
}

\author{
Wenchao Dan $\mathbb{D}^{1,2}$ Jinlei Liu $\mathbb{D}^{1,2}$ Xinyuan Guo $\mathbb{D}^{1,}{ }^{3}$ Boran Zhang $\mathbb{D}^{2}{ }^{2}$ Yi Qu $\mathbb{D},{ }^{1,2}$ \\ and Qingyong $\mathrm{He} \mathbb{( i D}^{1}$ \\ ${ }^{1}$ Department of Cardiology, China Academy of Chinese Medical Sciences Guanganmen Hospital, \\ No. 5, North Line Pavilion, Xicheng District, Beijing 100053, China \\ ${ }^{2}$ Graduate School of Beijing University of Chinese Medicine, Beijing 100029, China \\ ${ }^{3}$ Department of Radiation Therapy, Cancer Hospital Chinese Academy of Medical Sciences, \\ No. 17, South Panjiayuan, Chaoyang District, Beijing 100021, China \\ Correspondence should be addressed to Qingyong He; heqingyongg@163.com
}

Received 20 May 2020; Revised 16 August 2020; Accepted 21 October 2020; Published 18 November 2020

Academic Editor: Akhilesh K. Tamrakar

Copyright $\odot 2020$ Wenchao Dan et al. This is an open access article distributed under the Creative Commons Attribution License, which permits unrestricted use, distribution, and reproduction in any medium, provided the original work is properly cited.

\begin{abstract}
Background and Aim. Antineoplastic drug-induced cardiotoxicity (ADIC) becomes the second leading cause of death for tumor survivors after tumor recurrence and metastasis, and there may be great room for development in the future of traditional Chinese medicine (TCM). However, the theory of anticardiotoxicity of TCM has not yet formed a system. This study aimed to explore the material basis and the rule of TCM against ADIC based on network pharmacology and data mining. Methods. The targets of antineoplastic drugs with cardiotoxicity were obtained from the National Center for Biotechnology Information (NCBI) database, China national knowledge infrastructure (CNKI) database, and Swiss Target Prediction platform. Then, the cardiotoxicity-related targets were derived from the Gene Cards, Disgenet, OMIM, and DrugBank databases, as well as the drug of current clinical guidelines. The targets both in these two sets were regarded as potential targets to alleviate ADIC. Then, candidate compounds and herbs were matched via Traditional Chinese Medicine Systems Pharmacology (TCMSP) platform. Cytoscape3.7.1 was used to set up the target-compound-herb network. Molecular docking between core targets and compounds was performed with AutodockVina1.1.2. The rules of herbs were summarized by analyzing their property, flavor, and channel tropism. Results. Twenty-one potential targets, 332 candidate compounds, and 400 kinds of herbs were obtained. Five core targets including potassium voltagegated channel subfamily $\mathrm{H}$ member $2(\mathrm{KCNH} 2)$, cyclin-dependent kinase 1 (CDK1), matrix metalloproteinase 2 (MMP2), mitogen-activated protein kinase1 (MAPK1), and tumor protein p53 (TP53) and 29 core compounds (beta-sitosterol, quercetin, kaempferol, etc.) were collected. Five core herbs (Yanhusuo, Gouteng, Huangbai, Lianqiao, and Gancao) were identified. Also, the TCM against ADIC were mainly bitter and acrid in taste, warm in property, and distributed to the liver and lung meridians. Conclusion. TCM against ADIC has great potential. Our study provides a new method and ideas for clinical applications of integrated Chinese and western medicine in treating ADIC.
\end{abstract}

\section{Background}

Since the 1970s, there have been reports of cardiotoxic reactions caused by anthracyclines [1]. With the continuous enrichment of cancer treatment methods, the survival time of cancer patients has been greatly improved, and there are more and more reports of cardiac toxicity. According to statistics, about $51 \%$ of cancer patients die from recurrent disease, and about $33 \%$ of them die from heart disease, which is related to the increased incidence of coronary artery disease and valve heart disease [2]. At present, there are many research studies related to cardiotoxicity in tumor treatment drugs. For example, tyrosine kinase (TK) is a ubiquitous phosphorylase that controls many signaling pathways, such as VEGF and EGFR. Inhibition of this pathway by antibodies has become a new method of clinical 
treatment, which is accompanied by damage to heart function after inhibition of signal transduction. For example, sunitinib can inhibit more than 50 different kinases to play an antitumor effect, but at the same time, inhibiting AMPK affects the oxidative stress response and increases the chance of heart failure [3]. ERBB receptor antibody is a commonly used drug in the clinical treatment of breast cancer. At the same time of treatment, it inhibits the signal transduction of neuromodulin 1 (NRG-1), affects the metabolism of cardiomyocytes, and leads to the development of cardiac toxicity [4]. Cardiotoxic reactions caused by anthracycline antibiotics are more common. During doxorubicin chemotherapy, reactive oxygen species (ROS) production, DNA damage, and mitochondrial dysfunction can be caused, resulting in increased left ventricular wall pressure and decreased left ventricular ejection fraction (LVEF), arrhythmia and highly symptomatic congestive heart failure, and other adverse consequences [5]. Radiation therapy is widely used in cancer treatment, and it also causes heartrelated side effects during treatment. Its combination with anthracycline drugs also increases the risk of disease, which causes atherosclerosis, valve insufficiency, and other diseases [6].

At present, although the detection of certain biological markers can predict the impairment of cardiac function, such as troponin levels are associated with diastolic dysfunction caused by anthracyclines [7], the mechanism of most cardiac toxic drugs is still unclear. Only by using certain $\beta$-blockers, ACEI inhibitors, angiotensin receptor blockers, and other drugs for symptomatic treatment, the side effects of radiotherapy and chemotherapy can be alleviated [8]. The period of cardiotoxicity also has a great impact on tumor treatment. Early toxicity affects the progress of tumor treatment, and the tumor growth cannot be controlled in time, which leads to the deterioration of the condition; the late onset of toxicity often leads to the destruction of cardiac function, affecting the quality of life of patients. Moreover, the complexity of clinical symptoms increases the difficulty of distinguishing cardiac toxicity and cardiac dysfunction [9]. Therefore, an in-depth study of the mechanism of tumor cardiotoxicity can choose a more appropriate treatment approach for tumor patients and improve the quality of life of tumor patients.

TCM shows remarkable curative effects in clinical treatment of many diseases, and Chinese herbal medicine is a treasure trove of natural compounds, which have the advantages of wide source, multiple effective compounds, stable compound structure, and guaranteed safety. Reports have demonstrated that Xinmailong injection is effective for the treatment of cardiotoxicity caused by sequential chemotherapy of trastuzumab and anthracyclines in breast cancer, which could effectively improve the clinical symptoms of patients, attenuate myocardial injury, reduce blood viscosity, and inhibit expressions of serum IL- 6 and TNF- $\alpha$ [10]. It was confirmed that Danshen injection has particular preventive effects on cardiotoxicity caused by chemotherapy of pyrroxine or epirubicin, and the effect includes protecting the myocardium, relieving myocardial damage, and improving cardiac function [11]. However, there is less research on the Chinese herbs medicine against cardiac toxicity caused by antineoplastic drugs. A meta-analysis study showed that Wenxin grenules can prevent and reduce tachycardia caused by anthracyclines, but its efficacy in improving overall efficiency and preventing and reducing atrial premature beats, ventricular premature beats, atrial fibrillation, and SOD levels is not clear; meanwhile, Baoxinkang can protect myocardial SOD activity [12]. Therefore, it is of great value to further explore Chinese herbs medicine against cardiac toxicity caused by antineoplastic drugs and to develop related preparations. However, TCM and compound medicines have the characteristics of multicompound, multitarget, and multimechanism system regulation. If a single drug, single target, and single research idea are used for research, it is difficult to systematically reflect the intervention mechanism of TCM; as an emerging research discipline, Chinese medicine started late in this field, and no research has systematically summarized the rules of TCM against cardiac toxicity caused by antineoplastic drugs.

Network pharmacology is a combination of system pharmacology, multidirectional pharmacology, bioinformatics, data mining, and other multidisciplinary. By constructing a network to analyze the relationship between the various compounds and the key nodes in the network, it could show the material basis and mechanism of action of Chinese medicine or compound [13, 14]. Therefore, it is currently widely used in the research of the mechanism of action and new drug development of Chinese medicine and compound.

In this study, we used the method of network pharmacology, used the relevant targets of cardiac toxicity caused by antineoplastic drugs as entry points, and matched the relevant ligands and TCM. As a result, we systematically predicted and analyzed the complex relationship between the target, compound and TCM, meanwhile initially discussed the material basis and general rules, which could provide ideas and theoretical basis for the follow-up selection of TCM, theoretical discussion, new drug development, and clinical integration of traditional Chinese and Western medicine. The flow chart of this study is shown in Figure 1.

\section{Materials and Methods}

2.1. The Collection of Potential Targets for Herbs against Antineoplastic Drug-Induced Cardiotoxicity. The ADIC is mainly manifested as arrhythmia, heart failure, and ischemic heart disease [15]. To obtain relevant targets, cardiotoxicityrelated words, such as "arrhythmia," "heart failure," and "myocardial ischemia," were used as keywords to query the following electronic databases: Genecards database [16] (https://www.genecards.org/), OMIM database [17] (https:// omim.org/), DrugBank database [18], and Disgenet database [19] (http://www.disgenet.org). Results were merged, and duplicates were removed to obtain target group I. The sdf format files of antineoplastic drugs, including anthracyclines (doxorubicin (DOX), epirubicin, pirarubicin, aclarubicin, idarubicin, amrubicin, and daunorubicin), taxanes 


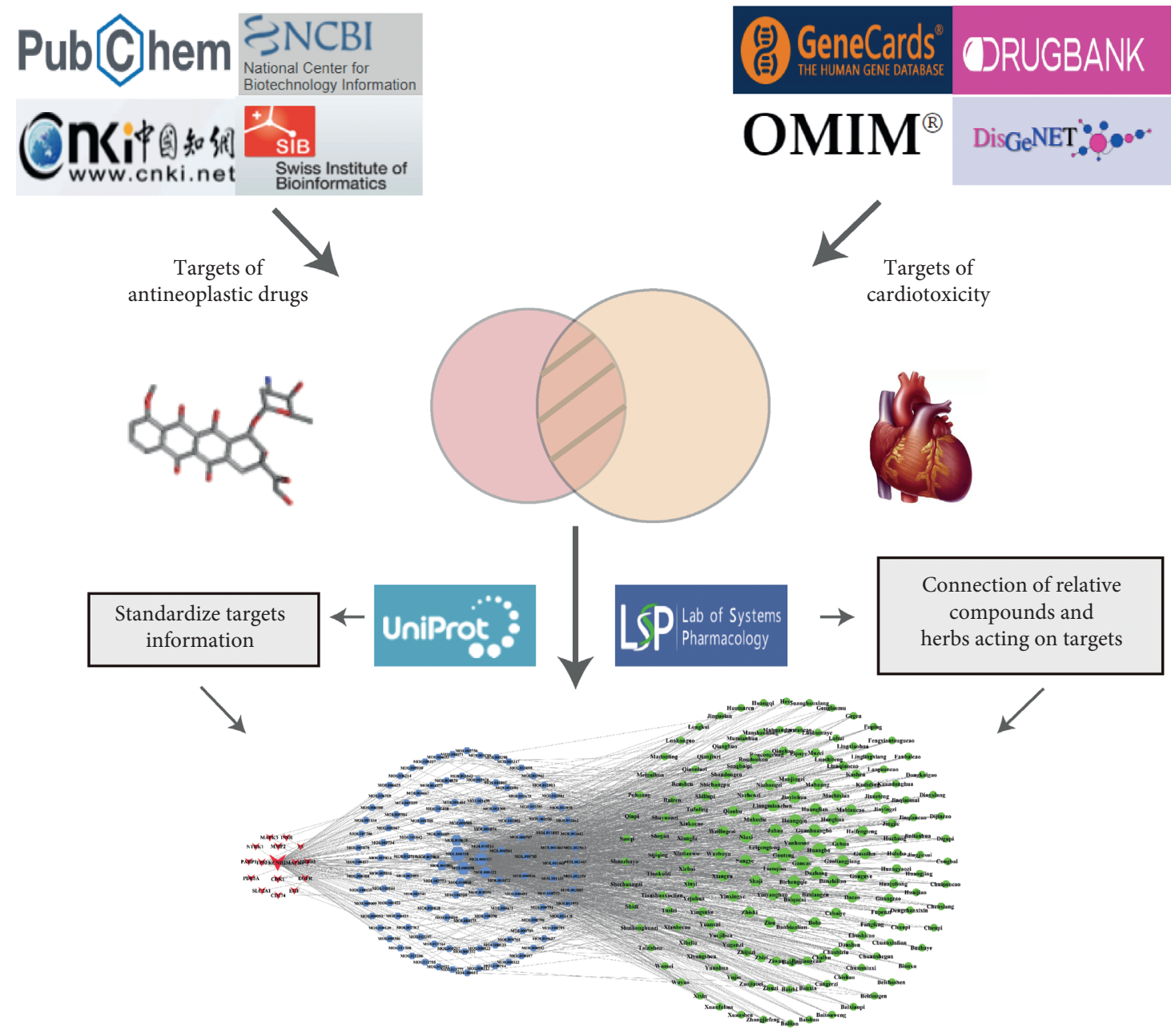

Targets-compounds-herbs network

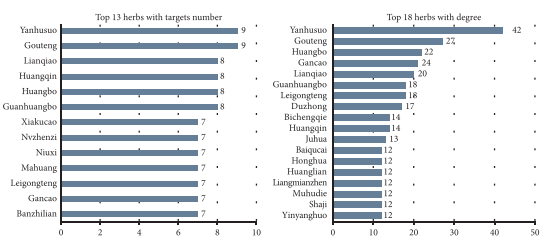

Potential core herbs

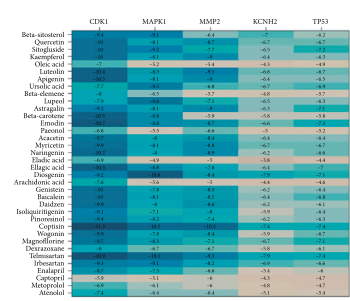

Molecular docking

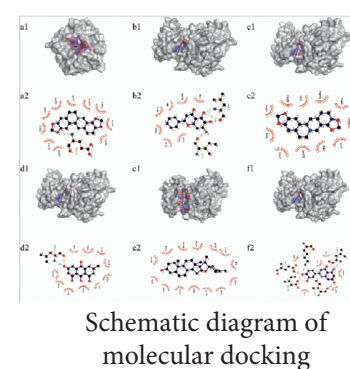

molecular docking

FIGURE 1: Whole framework based on network pharmacology.

(paclitaxel and docetaxel), and tyrosinase inhibitors, were derived from Pubchem, and used to predict compound targets via the Swiss TargetPredicition platform [20] (http:// www.swisstargetprediction.ch/). Then, we supplemented the results by consulting the literature about the targets related to human epidermal growth factor receptor 2 (HER-2) inhibitors and immune checkpoint blockade therapy. After removing the duplicate targets, we obtained target group II. The recommended drugs for ADIC were confirmed by consulting guidelines and consensus [21-23], and their targets were set as target group III. Then, the intersection of target group I and II were merged with target group III to get the final dataset of potential targets for TCM against ADIC. The information of these targets was standardized using the Uniprot database [24] (https://www.uniprot.org/).

\subsection{Candidate Compound Screening and Target-Compound} Network Construction. By searching the Traditional Chinese Medicine Systems Pharmacology (TCMSP) platform [25] (http://tcmspw.com/tcmsp.php), the compounds that can act on relevant targets are collected. According to the 
pharmacokinetic parameters (ADME) and the Lipinski rules [26], candidate compounds were screened. The specific screening criteria were oral availability $(\mathrm{OB}) \geq 30 \%$, drug likeness [27] $(\mathrm{DL}) \geq 0.18$, drug half-life $>4 \mathrm{~h}$, topological polar surface area $[28]<140$ angstroms, rotatable bonds number $[29] \leq 10$; and molecular weight $\leq 500 \mathrm{Da}$. Considering that the parameters provided by the TCMSP platform is predicted by the computer, part of the data may be inconsistent with the actual situation, and the deleted compounds were checked one by one to supplement the relevant active compounds after the initial screening. The revised compounds were regarded as candidate compounds. Along with these compounds, the potential targets were imported into Cytoscape3.7.2 software [30] to construct a targetcompound network and calculate the topology parameter (degree) of the nodes in the network. Core targets, compounds, and herbs were defined based on the degree.

\subsection{Herb Matching and Target-Compound-Herb Network} Construction. We collected herbs containing candidate compounds and constructed the compound-herb network. The "target-compound" network and the compound-herb network were combined through the merge function module in the Cytoscape 3.7.2 to establish the target-compound-herb network. Furthermore, we identified key nodes based on the degree of each node in the network and evaluated the strength of efficacy of the Chinese medicines and compounds in treating ADIC.

2.4. Characteristics of the Candidate Herbs. A frequency analysis was performed to summarize the rules of candidate herbs against ADIC. Characteristics under investigation included property, flavor, and channel tropism. Considering the heterogeneity of the information on characteristics from different sources, the priority order of standards we adopted in this study was Chinese Pharmacopoeia (2015) [31], Chinese Pharmacy ("Thirteenth Five-Year Plan" textbook) [32], and Chinese Dictionary of Clinical Medicine [33]. Herbs lacking of relevant information were deleted.

2.5. Molecular Docking of the Target-Compound. To assess the credibility of the connection between the target and the compound and identify new candidate herbs for ADIC treatment, molecular docking of the core compounds with core targets was carried out. Five targets with the highest degree in the "target-compound-herb" network were treated as receptors, and core compounds, as well as anti-ADIC drugs (atenolol, captopril, dexrazoxane, enalapril, irbesartan, metoprolol, and telmisartan) recommended by the guideline, were treated as ligands.

The crystal structure of the 5 proteins was downloaded from the Protein Data Bank (http://www.rcsb.org/pdb) and saved in a PDB format. The three-dimensional (3D) conformer structure of candidate compounds was downloaded from the PubChem database (https://pubchem.ncbi.nlm. nih.gov/) and saved in a SDF format that was subsequently converted to a PDB format with Open babel 2.4.1.
Ligands and receptors were prepared via AutoDock Tools (v.1.5.6) and PyMOL (v.2.3). The work of preparing receptors included deleting the original ligands and water molecules from the crystal structure of receptors, adding nonpolar hydrogens, and calculating Gasteiger partial charges. The flow of handling ligands contained applying energy minimization and assigning atomic charges and atom. All the prepared receptors and ligands were saved in a pdbqt format.

Then, the affinity indicating binding strength between the ligand and the target protein was evaluated with Autodock Vina (v.1.1.2). Affinity $<-4.25 \mathrm{kcal} / \mathrm{mol}$ means that ligands and receptors have possibility of combination, affinity $<-5.00 \mathrm{kcal} / \mathrm{mol}$ indicates good binding strength, and affinity $<-7.00 \mathrm{kcal} / \mathrm{mol}$ suggests satisfactory binding strength [34]. Pymol 2.3 and LigPlot 2.2 were used to visualize and analyze the docked conformations, and from the binding conformations of the docking results of each compound, the docking results with lower binding energy and better conformation were selected for presentation.

\section{Results}

3.1. Target Acquisition and Screening. A total of 84 antineoplastic drug-induced cardiotoxicity targets were obtained through Swisstarget Prediction and the literature, and 2,059 cardiotoxicity-related targets were obtained through the disease databases. A total of 40 targets were obtained from the intersection of the aforementioned two datasets. Additional 4 targets (ACE, AGTR1, AGTR2, and IFNAR1) of the anti-ADIC drugs (ACEI, ARB, and beta-blockers) recommended by clinical guidelines were also counted. Among these 44 targets, 21 were successfully matched to compounds that met the ADME and Lipinski screening criteria and were defined as potential targets for herbs against ADIC, as shown in Table 1.

\subsection{Candidate Compound Acquisition and Target-Compound} Network Construction. The potential targets were mapped by 13,729 small-molecule compounds in the TCMSP database, among which 332 candidate compounds met the ADME and Lipinski criteria. The target-compound network constructed by the potential targets and candidate compounds is shown in Figure 2. The network consisted of 353 nodes and 337 edges. The red nodes in the figure represent target points, the blue nodes represent compounds, the edges represent relations between two adjacent nodes, and the degrees represent the number of connected edges of nodes. The larger the degrees are, the greater regulatory roles the nodes play in the entire network. Figure 2 shows that $\mathrm{KCNH} 2$, CDK1, MMP2, MAPK1, and TP53 were the core targets, and their corresponding degrees were $239,16,15,14$, and 12 , respectively. Single targets with multiple compounds dominated the network but not vice versa.

3.3. Herb Acquisition and Target-Compound-Herb Network Construction. A total of 446 herbs were matched to the 332 compounds through the TCMSP database, 46 of which were 
TABLE 1: Information of potential targets.

\begin{tabular}{|c|c|c|c|}
\hline ID & Gene symbol & Uniprot ID & Protein name \\
\hline 1 & $\mathrm{ACE}$ & P12821 & Angiotensin-converting enzyme \\
\hline 2 & AGTR1 & P30556 & Type- 1 angiotensin II receptor \\
\hline 3 & CA1 & P00915 & Carbonic anhydrase I \\
\hline 4 & CD274 & Q9NZQ7 & Programmed cell death 1 ligand 1 \\
\hline 5 & CDK1 & P06493 & Cell division control protein 2 homolog \\
\hline 6 & EGF & P01133 & Proepidermal growth factor \\
\hline 7 & EGFR & P00533 & Epidermal growth factor receptor \\
\hline 8 & ERBB2 & P04626 & Receptor tyrosine-protein kinase erbB-2 \\
\hline 9 & F3 & P13726 & Tissue factor \\
\hline 10 & HDAC6 & Q9UBN7 & Histone deacetylase 6 \\
\hline 11 & IGFBP3 & P17936 & Insulin-like growth factor-binding protein 3 \\
\hline 12 & INSR & P06213 & Insulin receptor \\
\hline 13 & $\mathrm{KCNH} 2$ & Q12809 & Potassium voltage-gated channel subfamily $\mathrm{H}$ member 2 \\
\hline 14 & MAPK1 & P28482 & Mitogen-activated protein kinase 1 \\
\hline 15 & MAPK3 & P27361 & Mitogen-activated protein kinase 3 \\
\hline 16 & MMP2 & P08253 & $72 \mathrm{kDa}$ type IV collagenase \\
\hline 17 & NTRK1 & P04629 & High affinity nerve growth factor receptor \\
\hline 18 & PARP1 & P09874 & Poly [ADP-ribose] polymerase 1 \\
\hline 19 & PDE5A & O76074 & CGMP-specific $3^{\prime}, 5^{\prime}$-cyclic phosphodiesterase \\
\hline 20 & SLC2A1 & P11166 & Solute carrier family 2 , facilitated glucose transporter member 1 \\
\hline 21 & TP53 & P04637 & Cellular tumor antigen p53 \\
\hline
\end{tabular}

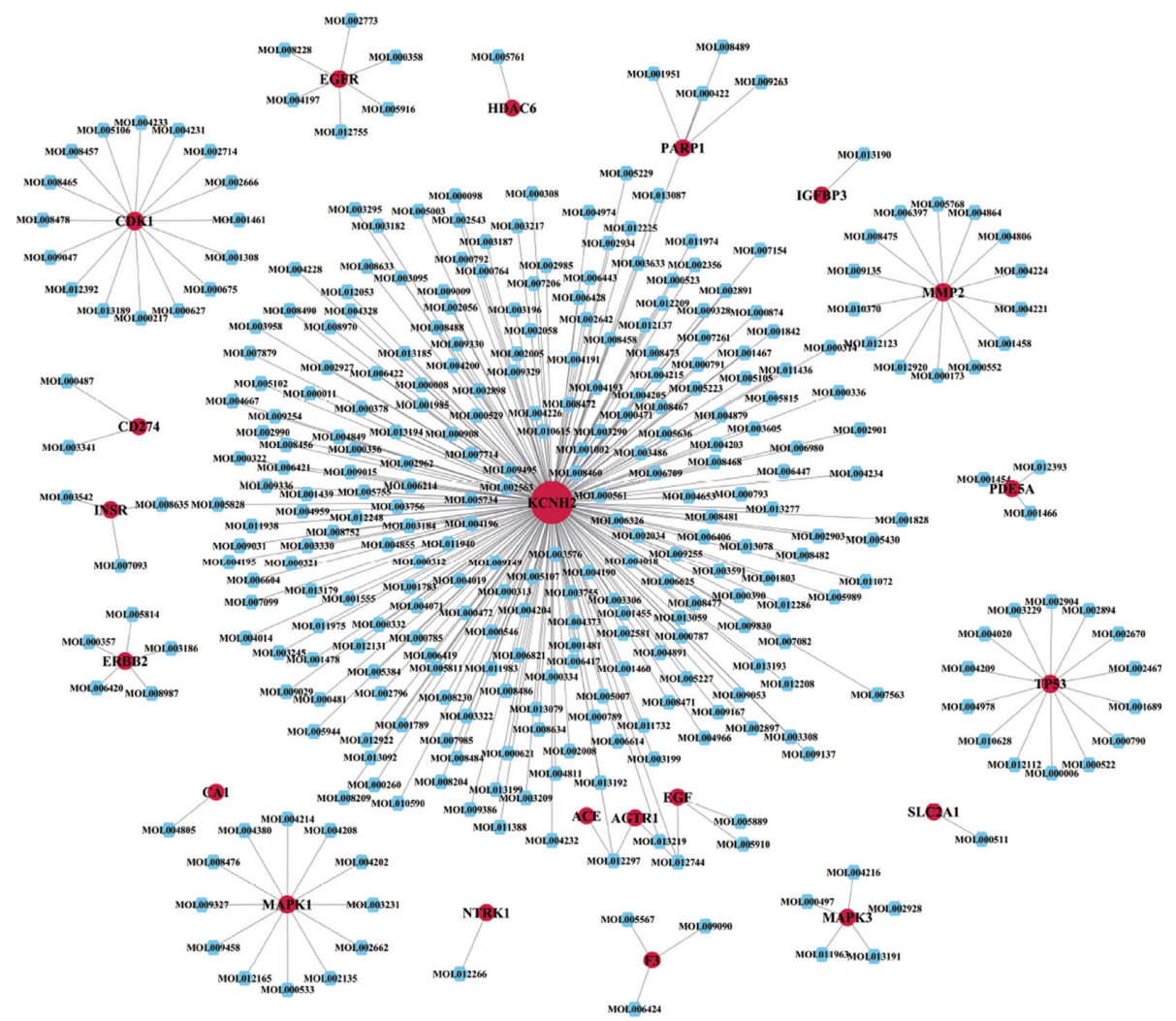

Figure 2: Target-compound network.

not included in the Chinese Pharmacopoeia (2015), Chinese Pharmacy ("Thirteenth Five-Year Plan" textbook), and Chinese Dictionary of Clinical Medicine, so we finally collected 400 herbs. Based on this, we constructed a compoundherb network. The network contained 732 nodes and 1,925 edges. Among them, the top 8 herb nodes with the highest degree were Yanhusuo, Gouteng, Huangbo, Gancao, Lianqiao, Guanhuangbo, Leigongteng, and Duzhong, and they contained $42,27,22,21,20,18,18$, and 17 candidate compounds, respectively. The targets of herbs were collected through the bridging of candidate compounds. The results showed that Yanhusuo, Gouteng, Lianqiao, Huangqin, 
Huangbo, Guanhuangbo, Xiakucao, and Nvzhenzi contained the most targets $(9,9,9,9,8,8,8$, and 8 , respectively). Therefore, we speculate that the abovementioned herbs have stronger regulatory effect on alleviating ADIC in the 400 herbs. The number of candidate compounds and targets of the herbs is shown in Figure 3. The median of the compound degree was 4 , and compounds with a degree greater than 8 were considered as potential core compounds. The top 5 compounds were beta-sitosterol, quercetin, daucosterol, kaempferol, and oleic acid. The remaining compounds are shown in Table 2.

In order to present the relation among potential targets, compounds, and herbs more concisely, our study reconstructed the target-compound-herb network by selecting herbs with degree $\geq 5$ and their related compounds and targets, as shown in Figure 4 . In the figure, the red nodes represented targets, the blue nodes represented compounds, and the green nodes represented herbs. The size of each node was positively correlated with the degree.

3.4. Properties, Tastes, and Meridian Tropism of Herbs. By analyzing the information of properties, tastes, and meridian tropism of the 400 herbs intervening ADIC, our results showed that herbs with bitter taste had the highest frequency, followed by acrid and sweet taste, and the proportion of the three tastes was $80.51 \%$; most herbs were warm. In terms of the meridian tropism, herbs belonging to liver meridian tropism accounted for the most proportion among the 400 herbs, which was $21.36 \%$, herbs belonging to lung meridian tropism accounted for $17.48 \%$, and herbs belonging to both liver and lung meridian tropism accounted for $38.83 \%$. The results are shown in Table 3 and Figure 5.

3.5. Molecular Docking. Twenty-nine core potential compounds were molecularly docked with 5 core targets including KCNH2 (PDBID: 6SYG), CDK1 (PDBID: 4Y72), TP53 (PDBID: 3Q05), MMP2 (PDBID: 1RTG), and MAPK1 (PDBID: 6SLG). A total of 145 pairs of receptor-ligand combinations were obtained, among which 79 combinations had affinity $<-7 \mathrm{kcal} / \mathrm{mol}$, accounting for $54.48 \%$. Twentyfive combinations were in the target-compound network, with the strongest binding in MMP2-coptisine $(-10.2 \mathrm{kcal} /$ mol) and the weakest in $\mathrm{KCNH} 2$-arachidonic acid $(-4.4 \mathrm{kcal} / \mathrm{mol})$. The average value of the 25 combinations was $-6.684 \mathrm{kcal} / \mathrm{mol}$, suggesting that the binding between the potential core targets and the compounds was strong.

The remaining 120 combinations were outside the target-compound network. The top 3 combinations with the highest affinity were CDK1-coptisine $(-11.3 \mathrm{kcal} / \mathrm{mol})$, CDK1-emodin (-10.7 kcal/mol), and MAPK1-diosgenin $(-10.6 \mathrm{kcal} / \mathrm{mol})$. There were 6 combinations with affinity $<-10.2 \mathrm{kcal} / \mathrm{mol}$. The docking strength of these 6 combinations exceeded that of the 25 combinations in the target-compound network. The docking results indicated that the number of potential compound-target combinations may be far greater than that of the compound-target combinations included in the TCMSP database, which shows that there are still a large number of connections between active TCM compounds and cardiotoxicity targets waiting to be further studied and excavated. The docking results can provide data support for future experimental screening of related TCM and compounds. The results are shown in Figure 6.

Comprehensively considering the molecular docking affinity value and the degree value in the target-compoundherb network, the results of docking with affinity $<-10 \mathrm{kcal} /$ mol in the network and those with the top 4 affinity outside the network were shown in the $3 \mathrm{D}$ and $2 \mathrm{D}$ format. It can be seen from the figure that each compound ligand is embedded in the active pocket of the receptor target and interacts with multiple residues of the target through hydrophobic interaction and hydrogen bond. The diagrams are shown in Figures $7(\mathrm{a}) \sim 7(\mathrm{~h})$.

\section{Discussion}

A number of reports have shown the potential of natural herbs in decreasing the cardiotoxic effects of chemotherapeutic agents on healthy cells, without negatively affecting their antineoplastic activity [35]. Subsequent improvement in heart function and quality of life may make chemotherapy more sustainable and reduce treatment-related mortality. Besides, most of TCM has anticancer effects with few side effects. Some physicians, thus, turned to use TCM as an alternative or complementary treatment to prevent and treat ADIC [36]. However, systematic studies of TCM intervention on ADIC are still insufficient. It is of great clinical significance to study the intervention mechanism of TCM against ADIC and to develop related pharmaceutical preparations on this basis.

4.1. Targets. In this study, 5 core targets (KCNH2, CDK1, MMP2, MAPK1, and TP53) were identified from the targetcompound-herb network. Dysfunction of KCNH2 activation will lead to the decrease of hERG current and a prolongation of the action potentials recorded in cardiomyocytes [37]. Dysfunction of KCNH2 expression is related to QT prolongation syndrome [38]. Inhibition of the KCNH2 potassium channel can lead to cardiotoxicity [39].

The overexpression of four regulatory factors of cell cycle including CDK1 was shown to efficiently induce cell division in postmitotic mouse, rat, and human cardiomyocytes, lead to a significant improvement in cardiac function after acute or subacute myocardial infarction, and improve heart function as indicated by significant improvement in the ejection fraction, stroke volume, and cardiac output.

MMP-2 can hydrolyze sarcomeric proteins in the heart, remodel the extracellular matrix, and lead to cardiotoxicity. Anthracycline drugs such as DOX can enhance the activity of MMP-2 in cardiomyocytes and may cause heart failure, so inhibition of MMP-2 activity can interfere with the cardiotoxicity of anticancer drugs [40].

DOX-induced mitochondrial reactive oxygen species (ROS) release was demonstrated to activate ERK-mediated heat shock factor 2 (HSF2) nuclear translocation and AT1 R upregulation, which caused DOX-damaged heart failure 


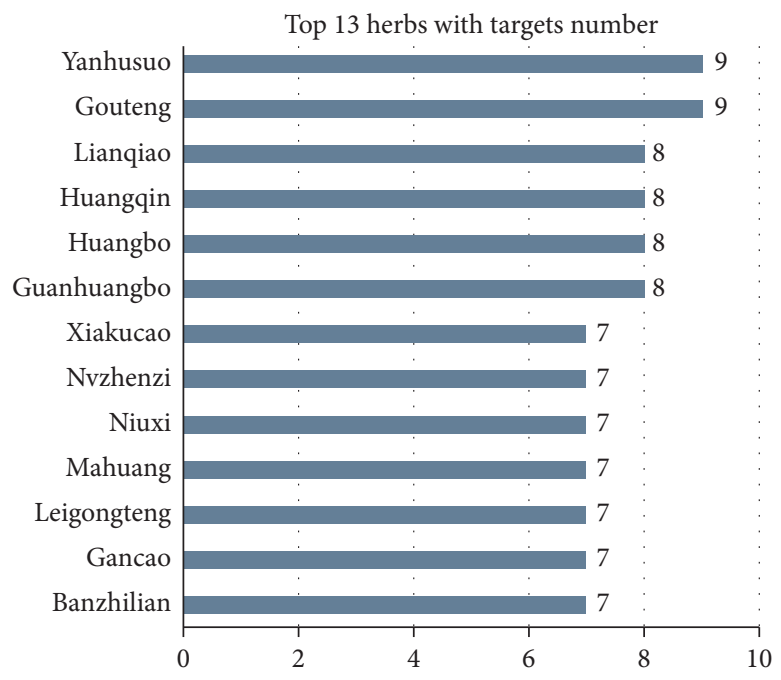

(a)

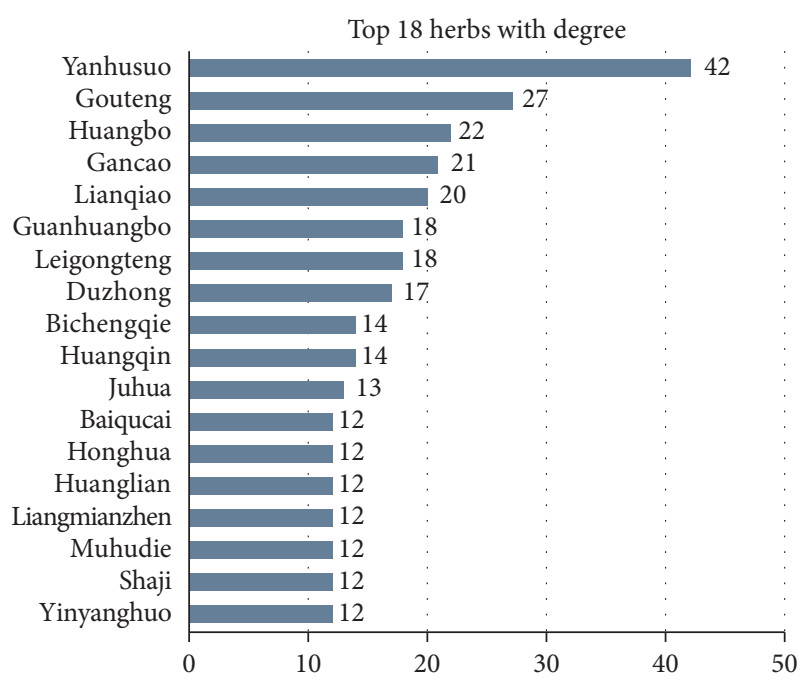

(b)

FIGURE 3: Distribution of candidate compounds and target sites in herbs.

TABLE 2: Information of candidate core compounds (degree $>8$ ).

\begin{tabular}{|c|c|c|c|c|c|}
\hline MolID & MolName & CAS & Degree & $\mathrm{OB}$ & $\mathrm{DL}$ \\
\hline MOL000358 & Beta-sitosterol & $83-46-5$ & 225 & 36.91391 & 0.75123 \\
\hline MOL000098 & Quercetin & $117-39-5$ & 174 & 46.43335 & 0.27525 \\
\hline MOL000357 & Sitogluside & $474-58-8$ & 170 & 20.63194 & 0.6241 \\
\hline MOL000422 & Kaempferol & $520-18-3$ & 122 & 41.88225 & 0.24066 \\
\hline MOL000675 & Oleic acid & $112-80-1$ & 109 & 33.12836 & 0.14243 \\
\hline MOL000006 & Luteolin & $491-70-3$ & 83 & 36.16263 & 0.24552 \\
\hline MOL000008 & Apigenin & $520-36-5$ & 75 & 23.06216 & 0.21306 \\
\hline MOL000511 & Ursolic acid & $77-52-1$ & 72 & 16.7749 & 0.75457 \\
\hline MOL000908 & Beta-elemene & $515-13-9$ & 45 & 25.63362 & 0.060519 \\
\hline MOL000356 & Lupeol & $545-47-1$ & 36 & 12.12076 & 0.77716 \\
\hline MOL000561 & Astragalin & $480-10-4$ & 35 & 14.02685 & 0.73616 \\
\hline MOL002773 & Beta-carotene & $7235-40-7$ & 28 & 37.18433 & 0.58358 \\
\hline MOL000472 & Emodin & $518-82-1$ & 28 & 24.39832 & 0.23916 \\
\hline MOL000874 & Paeonol & $552-41-0$ & 26 & 28.78724 & 0.039185 \\
\hline MOL001689 & Acacetin & $480-44-4$ & 20 & 34.97357 & 0.24082 \\
\hline MOL002008 & Myricetin & $529-44-2$ & 20 & 13.74833 & 0.31057 \\
\hline MOL004328 & Naringenin & $67604-48-2$ & 20 & 59.2939 & 0.21128 \\
\hline MOL001308 & Elaidic acid & $112-79-8$ & 17 & 33.12836 & 0.14262 \\
\hline MOL001002 & Ellagic acid & $476-66-4$ & 17 & 43.06456 & 0.43417 \\
\hline MOL000546 & Eiosgenin & $512-04-9$ & 14 & 80.87792 & 0.80979 \\
\hline MOL001439 & Arachidonic acid & $506-32-1$ & 13 & 45.57325 & 0.20409 \\
\hline MOL000481 & Genistein & $446-72-0$ & 13 & 17.93288 & 0.21384 \\
\hline MOL002714 & Baicalein & $491-67-8$ & 12 & 33.51892 & 0.20888 \\
\hline MOL000390 & Daidzein & $486-66-8$ & 11 & 19.44106 & 0.18694 \\
\hline MOL001789 & Isoliquiritigenin & $961-29-5$ & 11 & 85.3218 & 0.14805 \\
\hline MOL001842 & Pinoresinol & $487-36-5$ & 11 & 4.250519 & 0.5159 \\
\hline MOL001458 & Coptisine & $3486-66-6$ & 10 & 30.67185 & 0.85647 \\
\hline MOL000173 & Wogonin & $632-85-9$ & 10 & 30.68457 & 0.22942 \\
\hline MOL002891 & Magnoflorine & $2141 / 9 / 5$ & 9 & 0.479985 & 0.54735 \\
\hline
\end{tabular}

[41]. It is suggested that the activation of the ERK2 pathway of the mitogen-activated protein kinase (MAPK) family can protect cardiomyocytes from DOX-induced cardiotoxicity [42].

The wild-type p53 protein, which is encoded by the TP53 gene, plays an important role as a tumor suppressor in regulation of cell cycle arrest, DNA repair, and apoptosis. The association between p53 overexpression, mutations, and drug resistance has been reported in bladder, breast, ovarian, and other types of cancer. Mutation of p53 protein was indicated to decrease sensitivity to anthracycline treatments in human bladder TCC cells [43]. Reactivation of mutant 


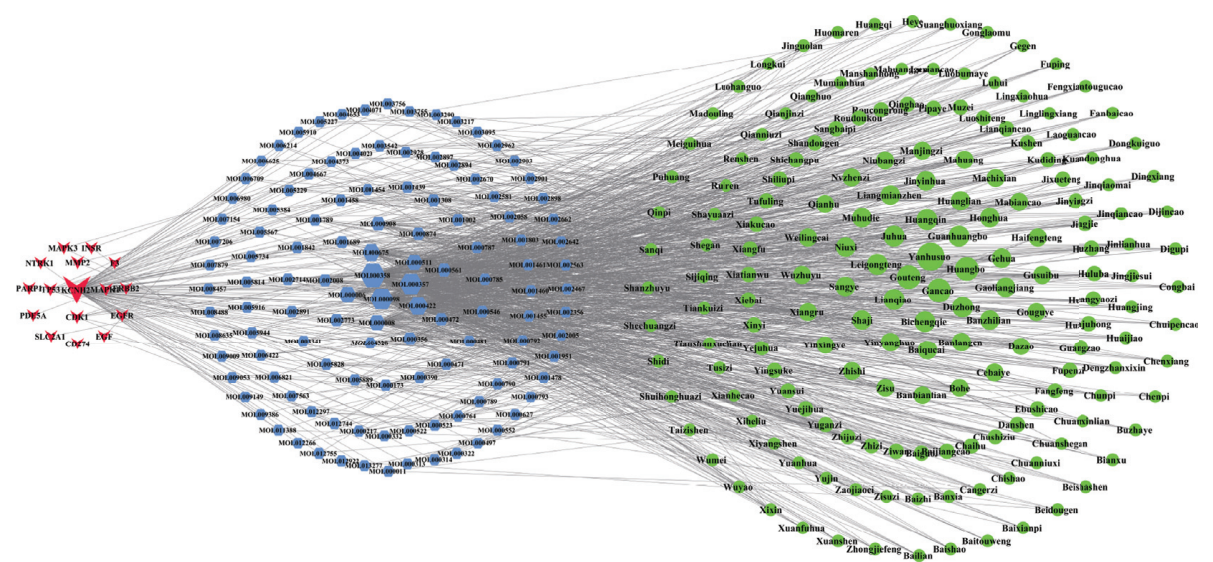

FIgURE 4: Target-compound-herb network (degree of herb $\geq 5$ ).

Table 3: Properties and tastes of herbs.

\begin{tabular}{|c|c|c|c|c|c|}
\hline Taste & Frequency & Proportion (\%) & Nature & Frequency & Proportion (\%) \\
\hline Bitter & 222 & 35.58 & Warm & 107 & 33.23 \\
\hline Acrid & 165 & 26.44 & Cold & 97 & 30.12 \\
\hline Sweet & 150 & 24.04 & Slight cold & 48 & 14.91 \\
\hline Astringent & 31 & 4.97 & Cool & 36 & 11.18 \\
\hline Sour & 29 & 4.65 & Slight warm & 24 & 7.45 \\
\hline Salty & 16 & 2.56 & Hot & 10 & 3.11 \\
\hline Light & 11 & 1.76 & & & \\
\hline
\end{tabular}

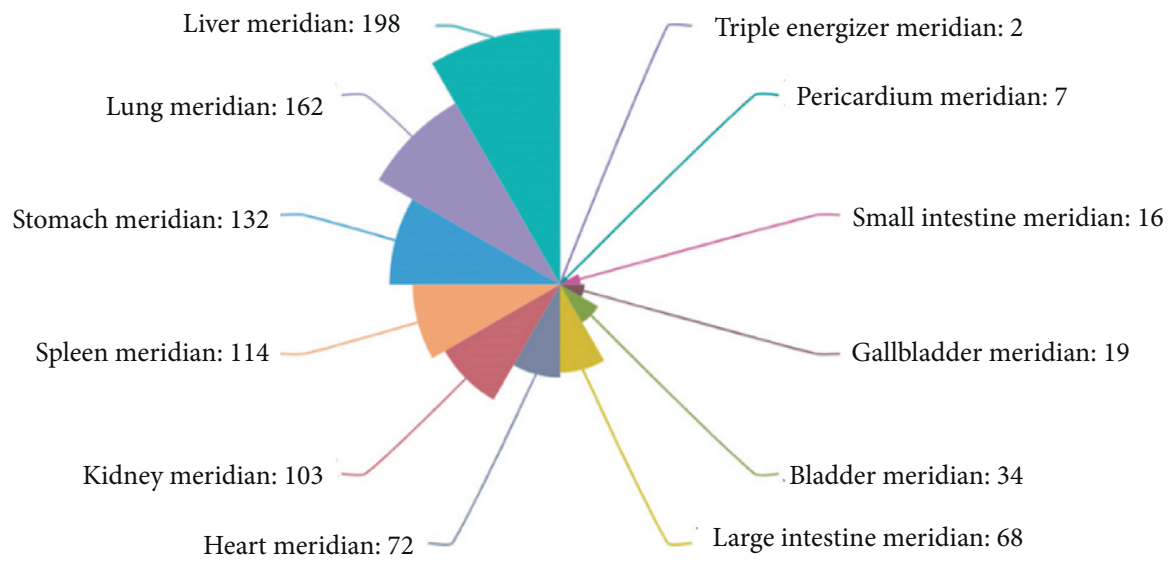

Figure 5: Meridian tropism of herbs.

p53 in those tumor cells may restore p53 tumor-suppressor function and sensitize mt-p53 cells to chemotherapy treatments [44]. Therefore, this study speculates that regulation of TP53 can enhance the sensitivity of tumor to anthracycline and other chemotherapy drugs, so as to reduce the dosage of these drugs and decrease their cardiotoxicity accordingly.

4.2. Compound. The core compounds obtained in this study included beta-sitosterol, quercetin, kaempferol, and oleic acid. Studies have shown that one of the core mechanisms of cardiotoxicity caused by anthracyclines is that ROS and reactive nitrogen species (RNS) will accumulate along with the metabolism of anthracyclines. A large amount of ROS and RNS may activate cytotoxic signals [45] in cardiomyocytes, leading to cardiac dysfunction through DNA damage, mitochondrial dysfunction, decreased antioxidant enzymes, and intracellular calcium homeostasis [46].

The intake of beta-sitosterol can enhance the storage of nonenzymatic antioxidants such as glutathione 1, vitamin C, and vitamin E [47]. Glutathione, the main antioxidative stress buffer in the cell, is widely used in cancer treatment. It mainly exists in the form of reduced glutathione (GSH) and oxidized glutathione (GSSG) in the human body. Experiments have shown that beta-sitosterol has a protective effect on the reduction of antioxidants containing GSH in colon 


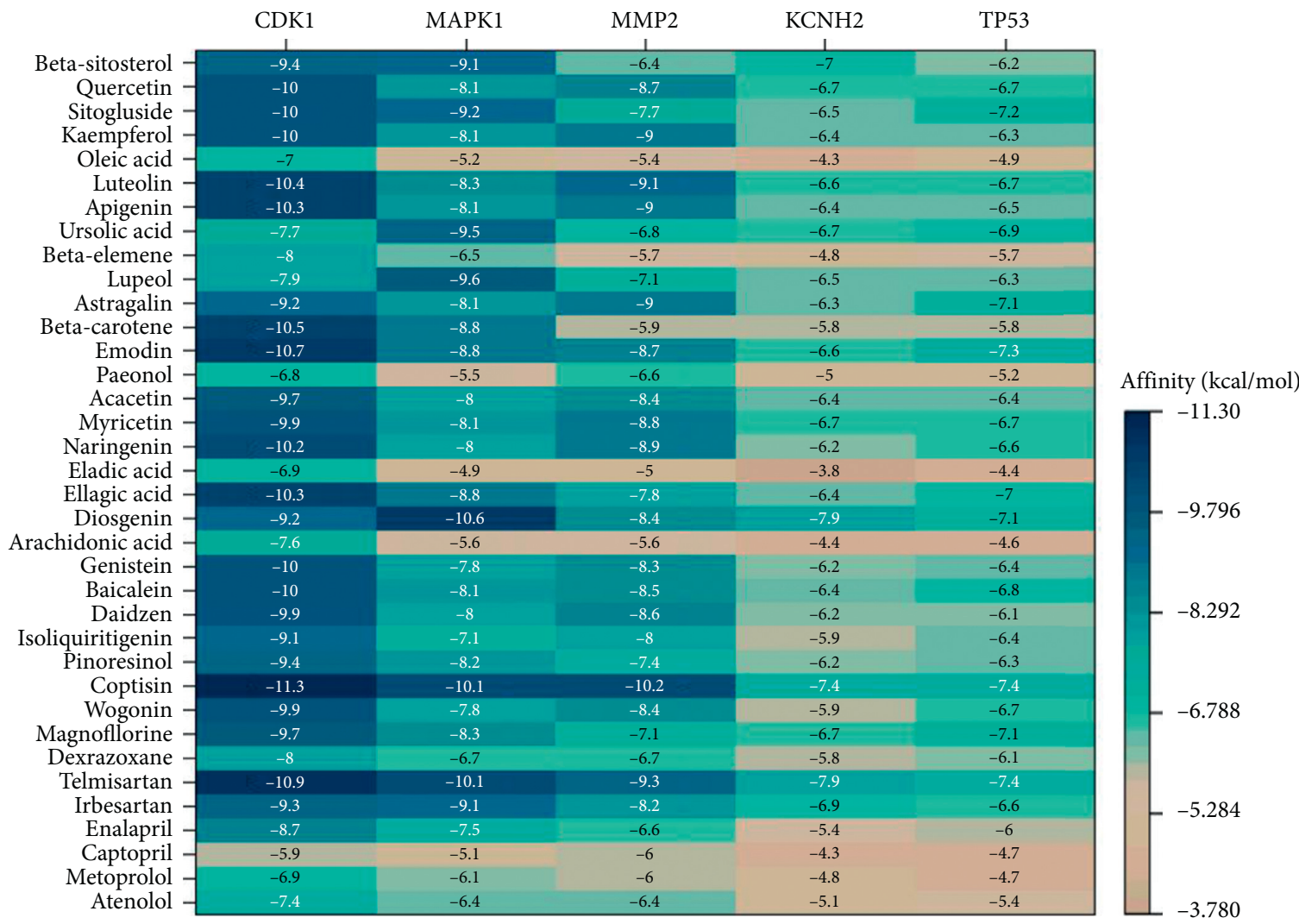

FIgURE 6: Results of molecular docking.

and liver tissues induced by 1, 2-dimethyl hydrazine [48]. Therefore, it is speculated that beta-sitosterol may mitigate ADIC by increasing the content of nonenzymatic antioxidants to compensate for the absence of antioxidant enzymes caused by anthracyclines.

Research showed that quercetin had significant anticancer activities against MCF-7, SKBR-3, and MDA-MB-231 human breast cancer cells and CT26 mouse colon cancer cells. It could also reduce the cardiotoxicity caused by DOX in the $\mathrm{Balb} / \mathrm{c}$ mice model [49].

Some studies demonstrated that kaempferol protected against DOX-induced cardiotoxicity by regulating the 14-3$3 \gamma$, MAPK, and ADMA/DDAHII/eNOS/NO pathway, inhibiting the activation of p53-mediated, mitochondriondependent apoptotic signaling, and improving mitochondrial function [50]. It has been reported that kaempferol dose dependently restored hemodynamic, left ventricular functions, decreased cardiac injury marker enzymes in serum, increased antioxidant levels, and reduced lipid peroxidation, TNF- $\alpha$ level, and apoptosis, so to alleviate cardiotoxicity [51].

Oleic acid has been found to have an excellent antioxidant effect. It was shown that oleic acid had a protective effect on myocardial mitochondrial injury induced by adrenaline in several aspects, such as inhibiting the elevated levels of lipid peroxidation and mitochondrial DNA damage, maintaining membrane intactness and the level of glutathione and keeping mitochondrial enzyme and membrane potential stable in rat experiments [52].
In conclusion, the potential core targets and compounds screened in this study were related to cardiotoxicity, which may play an important protective role in exploring the effects of TCM on ADIC.

4.3. Molecular Docking. The results of molecular docking showed that, in the target-compound network, the combination of MMP2-coptisine performed best, with affinity of $-10.2 \mathrm{kcal} / \mathrm{mol}$. There is considerable accumulating evidence suggesting a role of MMP-2, MMP-3, MMP-9 in promoting tumor cell invasion and infiltration $[53,54]$. The activation of the PI3K/Akt pathway associated with matrix metalloproteinases (MMPs) is essential for the growth and survival of cancer cells. Coptisine is a natural isoquinoline alkaloid. Studies have confirmed that coptisine has an improvement effect on cardiovascular disease in vitro and in vivo. It has strong antioxidant activity, helps maintain cell membrane integrity, improves mitochondrial respiratory dysfunction, and reduces cardiomyocyte apoptosis [55]. At the same time, it can protect cardiomyocytes from hypoxia or reoxidation-induced damage by inhibiting autophagy [56]. Simultaneously, coptisine can intervene in cancer through multiple ways. For instance, coptisine was found to induce apoptosis by strengthening the expression of the $67 \mathrm{kD}$ laminin receptor/cGMP pathway in various human hepatoma cells [57]. It can also affect a variety of apoptosisrelated targets, including Apaf-a, ROS, Bcl-2-XL, Bax, cytochrome c, Bid, AIF, XIAP, caspase- 3 , and caspase- 9 to 


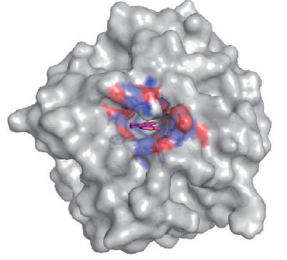

a1

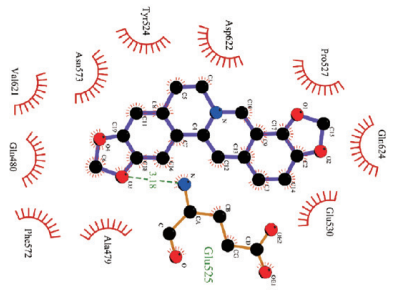

a2

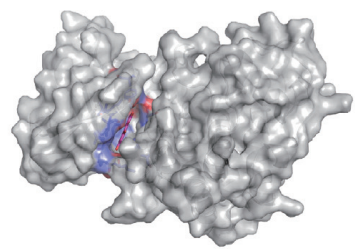

d1

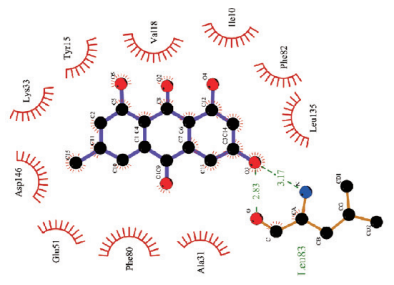

d2

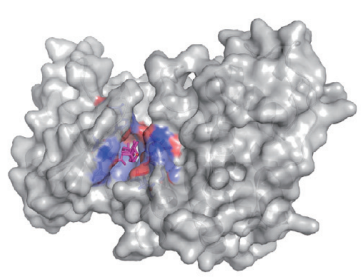

g1

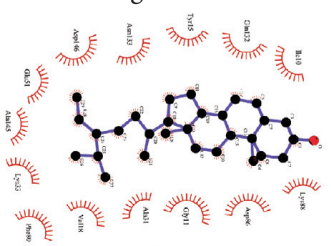

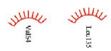

g2

$\leftrightarrow$ Ligand bond

- Hydrogen bond and its length

-. Non-ligand bond

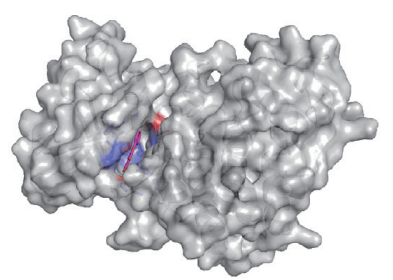

b1

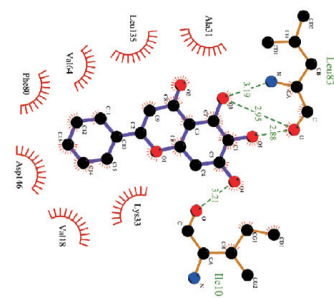

b2

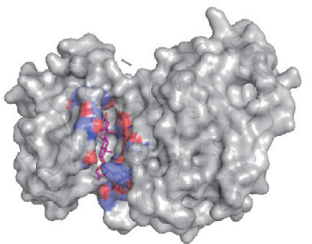

el

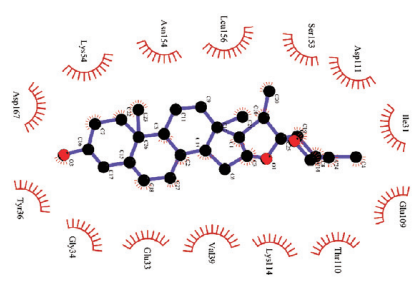

e2

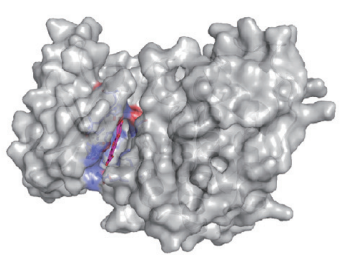

h1

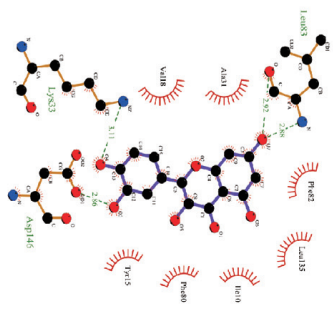

h2

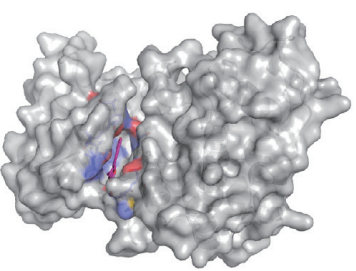

c1

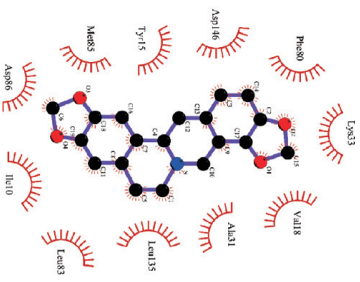

c2

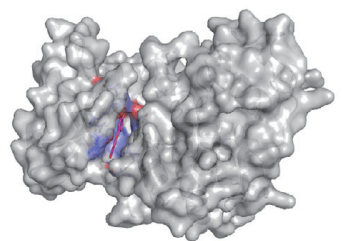

f1

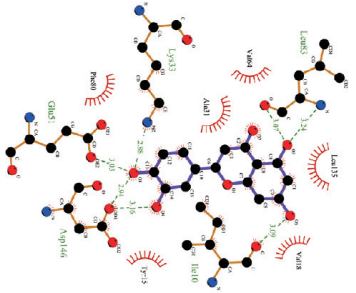

f2

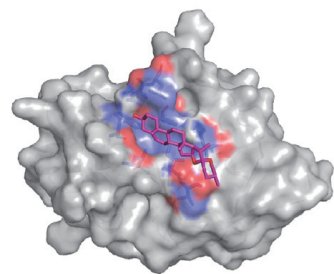

i1<smiles>CC(C)(C)CCCCC(C)(C)C</smiles>

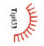

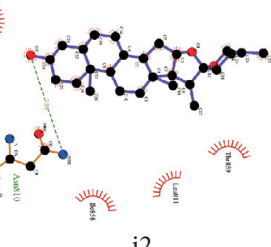

FIGURE 7: Molecular docking of compounds with core targets. (a) MMP2-coptsine; (b) CDK1-baicalein; (c) CDK1-coptsine; (d) CDK1emodin; (e) MAPK1-diosgenin; (f) CDK1-luteolin; (g) CDK1- $\beta$-sitosterol; (h) CDK1- quercetin; (i) KCNH2-diosgenin. 
inhibit the activity, adhesion, and migration of HCT-116 cells [58]. The abovementioned studies proved the reliability of this target-compound combination relationship in the network. We speculate that if coptisine is developed as a clinical drug, it may reduce the dose of cardiotoxic antitumor drugs, and at the same time, it may alleviate the oxidative stress response in cardiomyocytes caused by antitumor drugs, thereby improving myocardial function.

In addition to the target-compound network, the molecular docking results also showed that the six combinations of CDK1-coptisine, CDK1-emodin, MAPK1-diosgenin, CDK1-luteolin, CDK1-Apigenin, and CDK1-ellagicacid had good binding activity. Among them, emodin has been widely used in clinics because of its various pharmacological properties such as anticancer, antibacterial, antiviral, and liver protection properties [59]. Luteolin has been used clinically as a natural flavonoid. Clinical trials have shown that preparations containing luteolin have good anti-inflammatory effects [60], and in the treatment of glioma, combined with olaparib (PARP inhibition agent) or ionizing radiation treatment has certain clinical value [61]. Therefore, it can be regarded as one of the candidate drugs dealing with ADIC in future experimental or clinical researches.

4.4. Herbs. In order to better cooperate with tumor drug treatment, the selected herb can not only protect the heart but also has a certain antitumor effect. The core herbs obtained in this study included Yanhusuo, Gouteng, Huangbai, Lianqiao, and Gancao. Yanhusuo has the most therapeutic potential. Modern pharmacological research showed that corydalis had many functions such as antiarrhythmia, antimyocardial infarction, coronary artery expansion, antitumor, and antithrombosis functions [62], and the extract from corydalis had a protective effect on myocardial ischemia-reperfusion injury, which was closely associated with the inhibition of myocardial apoptosis through modulation of the Bcl-2 family [63]. Tetrahydropalmatine (THP), an effective compound of corydalis, with the analgesic effects on bone cancer pain may underlie the inhibition of microglial cell activation and the increase in proinflammatory cytokine. Meanwhile, THP can reverse the ventricular tachycardia induced by drugs in rabbits and exert an antiarrhythmia effect [64].

Alkaloid extracted from Gouteng may intervene in tachycardia and arrhythmia through blocking the calcium channel and opening the potassium channel [65]. Isorhynchophylline, extracted from Gouteng, has the potential of inhibiting cardiac hypertrophy through Nrf2 nuclear translocation and the MAPK pathway [66].

Huangbai has showcased a wide range of pharmacological effects, including anticancer, anti-inflammatory, and antimicrobial effects. The compound ((21S, 23R) epoxy-24hydroxy-21 $\beta, 25$-diethoxy) tirucalla-7-en-3-one has a relatively strong effect as adriamycin against four tumors. Polysaccharides from an aqueous extract of phellodendri act on cell-mediated stimulation and humoral immunity instead of tumor cell inhibition to exert tumoricidal activity [67]. Meanwhile, Huangbai has an antiarrhythmic effect [68].
The main components of Lianqiao suspense are phillyrin, forsythiaside, chlorogenic acid, and so on. Phillyrin can improve heart function by regulating the myocardial contractility index and reduce myocardial fibrosis after myocardial infarction, which showed an effect similar to that of enalapril [69]. Forsythiaside can effectively prevent hydrogen peroxideinduced mitochondrial membrane potential depolarization. Cytochrome $C$, caspase- 9 , and caspase- 3 are markers of mitochondrial-dependent apoptotic pathways that induce cell death, which is an oxidative stress reaction. Forsythiaside can effectively prevent this reaction induced by hydrogen peroxide [70]. Chlorogenic acid (CGA) attenuates TNF- $\alpha$-induced cardiomyocyte apoptosis by inhibiting JNK [71]. Li et al. found that CGA could inhibit isotype-induced cardiac hypertrophy through blocking the NF- $\kappa \mathrm{B}$ signaling pathway [72]. CGA can significantly reverse the decline in cardiac mitochondrial membrane potential induced by TNF- $\alpha$. Therefore, it is suggested that inhibiting TNF- $\alpha$ may help maintain the stability of mitochondrial membrane potential and prevent adriamycininduced cardiotoxicity in isolated rat hearts [73].

Gancao has protective effects on the heart, and the hydrolysates of its main components glycyrrhizin is glycyrrhetinic acid, which has the effect of intervening the permeability transition (MPT) of rat heart mitochondria [74]. The extract of Gancao can maintain the integrity of the cell membrane, improve lipid homeostasis, and stabilize actin. Gancao extract can reduce ROS levels and repair antioxidant status, thus helping to repair DNA damage and mitochondrial function. The SIRT-1-mediated pathway and its downstream activator PPARS are important for the maintenance of cell function. Glycyrrhiza extract could restore nuclear SIRT-1 and PPAR- $\gamma$ levels in $\mathrm{H} 9 \mathrm{C} 2$ cardiomyocytes reduced by DOX interference [75]. It is concluded that Gancao extract can antagonize the cardiotoxicity induced by DOX and maintain the normal function of cardiac myocytes.

The herbs mentioned above may have the potential of mitigating ADIC; meanwhile, some of them have extra beneficial effects on cancer patients. This will provide an alternative scheme for related trials on treating ADIC with TCM. At the same time, the information of herbal taste and meridian tropism can lay a certain foundation for the theoretical discussion and research of TCM on ADIC in the future. Herbs with multiple targets might be of great importance due to their potential broad-spectrum effects against ADIC. A combination of herbs containing various compounds corresponding to multiple targets might be an alternative strategy. The obtained core herbs and compounds can provide reference for the prescription exploration in integrating traditional Chinese and Western medicine to treat ADIC.

\section{Strengths and Limitations}

The advantage of this study lies in the use of network pharmacology, data mining, and molecular docking methods to obtain herbs and compounds that can potentially resist ADIC from a large number of TCM, which can save economic cost and time in the future study. Meantime, many rare diseases still lack effective means, and the efficacy of some common diseases is not ideal. Chinese medicine may have 
certain advantages, but screening out the ideal herb is very time consuming and costly, such as artemisinin. The method used in this research can provide a reference for similar research to narrow the selection scope. The deficiency of this study is that we focus on the relation among targets, compounds, and herbs, but the efficacy of herbs between the targets and the compounds and the compound content of each herb have not been fully discussed. There is a wide variety of antitumor drugs, and new drugs are emerging one after another. The drugs selected in this study came from the existing literature. However, some new drugs with potential cardiotoxicity are not included because of insufficient clinical evidence and no relevant reports have been seen so far. Thus, we will continue to follow-up related progress in the future.

\section{Conclusions}

This study constructed a target-compound-herb network using ADIC-related targets from multiple databases and compounds and herbs matched from the TCMSP platform. Core targets (KCNH2, CDK1, and MMP2), compounds (betasitosterol, quercetin, and kaempferol), and herbs (Yanhusuo, Gouteng, Huangbai, Lianqiao, and Gancao) that may be helpful in treating ADIC were identified from the network. The binding activity between the core targets and the core compounds were strong, verifying the reliability of their connection beyond the network. Most of the candidate herbs were bitter, acrid, and warm, belonging to liver and lung meridians. These conclusions provide new ideas for the screening of herbs that can intervene ADIC in an advanced, evidence-based, and systematic way, and also provide reference for the prescription combining TCM and Western medicine.

\section{Abbreviations}

$\begin{array}{ll}\text { TCM: } & \text { Traditional Chinese medicine } \\ \text { TCMSP: } & \text { Traditional Chinese Medicine Systems } \\ & \text { Pharmacology } \\ \text { DL: } & \text { Drug likeness } \\ \text { OB: } & \text { Oral availability } \\ \text { HL: } & \text { Drug half-life } \\ \text { TPSA: } & \text { Topological polar surface area } \\ \text { RBN: } & \text { Rotatable bonds number } \\ \text { MW: } & \text { Molecular weight } \\ \text { PDB: } & \text { Protein Data Bank } \\ \text { 3D: } & \text { Three dimensional } \\ \text { KCNH2: } & \text { Potassium voltage-gated channel subfamily H } \\ & \text { member 2 } \\ \text { CDK1: } & \text { Cyclin-dependent kinase 1 } \\ \text { MMP2: } & \text { Matrix metalloproteinase 2 } \\ \text { MAPK1: } & \text { Mitogen-activated protein kinase1 } \\ \text { MAPK: } & \text { Mitogen-activated protein kinase } \\ \text { TP53: } & \text { Tumor protein p53 } \\ \text { TK: } & \text { Tyrosine kinase } \\ \text { VEGF: } & \text { Vascular endothelial growth factor } \\ \text { EGFR: } & \text { Epidermal growth factor receptor } \\ \text { AMPK: } & \text { Adenosine 5'-monophosphate- (AMP-) } \\ & \text { activated protein kinase }\end{array}$

ERBB2: Human epidermal growth factor receptor (HER-2)

NRG-1: $\quad$ Neuromodulin 1

ROS: $\quad$ Reactive oxygen species

LVEF: Left ventricular ejection fraction

ACEI: Angiotensin-converting enzyme inhibitor

SOD: $\quad$ Superoxide dismutase

ARB: $\quad$ Angiotensin receptor blocker

hERG: Human Ether-a-go-go Related Gene

EF: $\quad$ Ejection fraction

ERK1/2: Extracellular regulatory protein kinase1/2

AT1 R: Angiotensin receptor1

DOX: Doxorubicin

TCC cells: Transitional cell carcinoma

mt-p53: $\quad$ Mutation of p53

RNS: $\quad$ Reactive nitrogen species

GSH: Glutathione

GSSG: Oxidized glutathione

DMH: $\quad$ 1: 2-dimethy lhydrazine

TNF- $\alpha$ : Tumor necrosis factor- $\alpha$

ADMA: Asymmetric dimethylarginine

DDAH II: Dimethylarginine dimethylamine hydrolase II

eNOS: $\quad$ Endothelial nitric oxide synthase

NO: $\quad$ Nitric oxide

MCF-7: Human breast cancer cells MCF-7

SKBR-3: Human breast cancer cells SKBR-3

MDA-MB- Human breast cancer cells MDA-MB-231

231:

CT26: $\quad$ Mouse colon cancer cells CT26

FoxO1: $\quad$ Forkhead box O1

NFATc3: Nuclear factor of activated T-cells,

PI3K/Akt: $\quad \begin{aligned} & \text { cytoplasmic, calcineurindependent } 3 \\ & \text { Phosphatidylinositol } 3 \text { kinase / protein kinase }\end{aligned}$

MMPs: $\quad$ Matrix metalloproteinases

cGMP: $\quad$ Cyclic guanosine monophosphate

Apaf-a: Apoptotic protease activating factor-a

Bax: $\quad$ BCL2-associated X

BID: $\quad$ BH3-interacting domain death agonist

AIF: $\quad$ Apoptosis inducing factor

XIAP: $\quad \mathrm{x}$-linked inhibitor of apoptosis protein

HCT-116: Human colon cancer Cell HCT-116

PARP: $\quad$ Poly ADP-ribose Polymerase

IR: Ionizing radiation

Bcl-2: b-cell lymphoma-2

THP: Tetrahydropalmatine

VT: Ventricular tachycardia

Nrf2: $\quad$ Nuclear factor E2-related factor 2

CGA: Chlorogenic acid.

\section{Data Availability}

The datasets used and/or analyzed during the current study are available from the corresponding author on reasonable request.

\section{Conflicts of Interest}

All authors declare that there are no conflicts of interest. 


\section{Authors' Contributions}

DWC and LJL contributed equally to this paper, and they designed the study. Experimental work and data collection were conducted by DWC and LJL. GXY and ZBR analyzed and interpreted the data. DWC and LJL drafted the manuscript. HQY and GXY provided critical comments and revised the manuscript. ZBR and QY helped perform the analysis with constructive discussions. All authors read and approved the final version of the manuscript.

\section{Acknowledgments}

The authors gratefully acknowledge the contribution of Jianliang Li and Jianbo Guo (Beijing University of Chinese Medicine) in the study design and guidance. This study was supported by the project Beijing Science and Technology Rising Star (Z181100006218035). The present study was supported by the National Natural Science Foundation of China (81202803) and the National Administration of TCM Project Fund (G2T-FJS-2019-203). The funders had no role in study design, data collection and analysis, decision to publish, or preparation of the manuscript.

\section{References}

[1] D. D. Von Hoff, "Risk factors for doxorubicin-lnduced congestive heart failure," Annals of Internal Medicine, vol. 91, no. 5, pp. 710-717, 1979.

[2] H. A. Preti, "Points to remember: practical pearls in oncocardiology," Methodist DeBakey Cardiovascular Journal, vol. 11, no. 4, p. 254, 2015.

[3] B. Sherry-Ann, R. C. Jordan, and H. Joerg, "Precision cardiooncology: a systems-based perspective on cardiotoxicity of tyrosine kinase inhibitors and immune checkpoint inhibitors," Journal of Cardiovascular Translational Research, vol. 13, no. 3, pp. 402-416, 2020.

[4] G. D'Uva, A. Aharonov, M. Lauriola et al., "ERBB2 triggers mammalian heart regeneration by promoting cardiomyocyte dedifferentiation and proliferation," Nature Cell Biology, vol. 17, no. 5, pp. 627-638, 2015.

[5] E. A. Pinheiro, T. Magdy, and P. W. Burridge, "Human in vitro models for assessing the genomic basis of chemotherapy-induced cardiovascular toxicity," Journal of Cardiovascular Translational Research, vol. 13, no. 3, pp. 377-389, 2020.

[6] S. Schultz-Hector and T. Klaus-Rüdiger, "Radiation-induced cardiovascular diseases: is the epidemiologic evidence compatible with the radiobiologic data?" International Journal of Radiation Oncology, Biology, Physics, vol. 67, no. 1, pp. 10-18, 2007.

[7] C. Cardinale, B. Giulia, M. Beggiato, A. Colombo, and C. M. Carlo, "Strategies to prevent and treat cardiovascular risk in cancer patients," Seminars in Oncology, vol. 40, no. 2, pp. 186-198, 2013.

[8] S. Seicean, A. Seicean, J. C. Plana, G. T Budd, and M. H. Thomas, "Effect of statin therapy on the risk for incident heart failure in patients with breast cancer receiving anthracycline chemotherapy: an observational clinical cohort study," Journal of the American College of Cardiology, vol. 60, no. 23, pp. 2384-2390, 2012.

[9] M. W. Bloom, C. E. Hamo, D. Cardinale et al., "Cancer therapy-related cardiac dysfunction and heart failure: Part 1: definitions, pathophysiology, risk factors, and imaging," Circulation Heart Failure, vol. 9, no. 1, Article ID e2661, 2016.

[10] Y. Tiezhu, X. Zhihong, M. Jing-tao, and Z. Ning, "Protection effect of Xinmailong injection in treating breast cancer patients with myocardial damage caused by sequential chemotherapy of protection effect of Xinmailong injection in treating breast cancer patients with myocardial damage caused by sequential chemotherapy of trastuzumab and anthracyclines," Chinese Journal of Experimental Traditional Medical Formulae, vol. 22, no. 6, pp. 154-158, 2016.

[11] Z. Xianjin, S. Lianxun, and C. Liuzhong, "Research on effects of danshen injection in preventing cardiotoxicity induced by chemotherapy of pyrroxine or epirubicin," Evaluation and Analysis of Drug-Use in Hospitals of China, vol. 18, no. 4, pp. 511-513, 2018.

[12] Z. Qin, Y. Xinyu, Z. Xiaoyu, and S. Hongcai, "Efficacy of traditional Chinese medicine for arrhythmia caused by anthracyline drugs: a systematic review," Chinese Journal of Evidence-Based Medicine, vol. 18, no. 6, pp. 604-609, 2018.

[13] W. Zhang, Y. Huai, Z. Miao, A. Qian, and Y. Wang, "Systems pharmacology for investigation of the mechanisms of action of traditional Chinese medicine in drug discovery," Frontiers in Pharmacology, vol. 10, p. 743, 2019.

[14] R. Zhang, X. Zhu, H. Bai, and K. Ning, "Network pharmacology databases for traditional Chinese medicine: review and assessment," Frontiers in Pharmacology, vol. 10, p. 123, 2019.

[15] K. Athanasios, A. Ntalianis, E. Repasos, E. Kastritis, M.-A. Dimopoulos, and I. Paraskevaidis, "Cardio-oncology: a focus on cardiotoxicity," European Cardiology, vol. 13, no. 1, pp. 64-69, 2018.

[16] S. Marilyn, D. Irina, A. Justin et al., "GeneCards Version 3: the human gene integrator," Database The Journal of Biological Databases and Curation, vol. 2010, p. 20, 2010.

[17] J. S. Amberger, C. A. Bocchini, S. François, A. F. Scott, and H. Ada, "OMIM.org: online mendelian inheritance in man (OMIM $\left.{ }^{\circledR}\right)$, an online catalog of human genes and genetic disorders," Nucleic Acids Research, vol. 43, no. D1, pp. D789-D798, 2015.

[18] D. S. Wishart, Y. D. Feunang, A. C. Guo et al., "DrugBank 5.0: a major update to the DrugBank database for 2018," Nucleic Acids Research, vol. 46, no. D1, pp. D1074-D1082, 2017.

[19] J. Piñero, J. M. Ramírez-Anguita, J. Saüch-Pitarch et al., "The DisGeNET knowledge platform for disease genomics: 2019 update," Nucleic Acids Research, vol. 48, no. D1, pp. D845-D855, 2020.

[20] A. Daina, O. Michielin, and V. Zoete, "SwissTargetPrediction: updated data and new features for efficient prediction of protein targets of small molecules," Nucleic Acids Research, vol. 47, no. W1, pp. W357-W364, 2019.

[21] S. H. Armenian, C. Lacchetti, and A. Barac, "Prevention and monitoring of cardiac dysfunction in survivors of adult cancers: American society of clinical oncology clinical practice guideline," Journal of Clinical Oncology, vol. 35, no. 8, pp. 893-911, 2017.

[22] S. A. Virani, S. Dent, C. Brezden-Masley et al., "Canadian cardiovascular society guidelines for evaluation and management of cardiovascular complications of cancer therapy," Canadian Journal of Cardiology, vol. 32, no. 7, pp. 831-841, 2016.

[23] J. L. Zamorano, P. Lancellotti, D. Aboyans et al., "2016 ESC Position Paper on cancer treatments and cardiovascular toxicity developed under the auspices of the ESC committee 
for practice guidelines," European Heart Journal, vol. 37, no. 36, pp. 2768-2801, 2016.

[24] A. Rolf, B. Amos, C. H. Wu et al., "UniProt: the universal protein knowledgebase," Nucleic Acids Research, vol. 32, no. suppl_1, 2004.

[25] J. Ru, P. Li, J. Wang et al., "TCMSP: a database of systems pharmacology for drug discovery from herbal medicines," Journal of Cheminformatics, vol. 6, no. 1, pp. 1-6, 2014.

[26] C. A. Lipinski, F. Lombardo, B. W. Dominy, and P. J. Feeney, "Experimental and computational approaches to estimate solubility and permeability in drug discovery and development settings," Advanced Drug Delivery Reviewsvol, vol. 23, no. 1-3, pp. 3-25, 2012.

[27] W. Tao, X. Xu, X. Wang et al., "Network pharmacology-based prediction of the active ingredients and potential targets of Chinese herbal Radix Curcumae formula for application to cardiovascular disease," Journal of Ethnopharmacology, vol. 145, no. 1, pp. 1-10, 2013.

[28] P. Ertl, B. Rohde, and P. Selzer, "Fast calculation of molecular polar surface area as a sum of fragment-based contributions and its application to the prediction of drug transport properties," Journal of Medicinal Chemistry, vol. 43, no. 20, pp. 3714-3717, 2000.

[29] D. F. Veber, S. R. Johnson, H.-Y. Cheng, B. R. Smith, K. W. Ward, and K. D. Kopple, "Molecular properties that influence the oral bioavailability of drug candidates," Journal of Medicinal Chemistry, vol. 45, no. 12, pp. 2615-2623, 2002.

[30] D. Otasek, J. H. Morris, J. Bouças, A. R. Pico, and B. Demchak, "Cytoscape Automation: empowering workflow-based network analysis," Genome Biology, vol. 20, no. 1, p. 185, 2019.

[31] NP Committee, Chinese Pharmacopoeia, China Medical Science and Technology Press, Beijing, China, 2015.

[32] Z. Gansheng, Chinese Pharmacy, China Press of Traditional Chinese Medicine, Beijing, China, 2016.

[33] P. Cheng, Chinese Dictionary of Clinical Medicine, China Medical Science and Technology Press, Beijing, China, 2018.

[34] H. Kun-Yi, G. Samik, and K. Hiroaki, "Combining machine learning systems and multiple docking simulation packages to improve docking prediction reliability for network pharmacology," PLoS One, vol. 8, no. 12, Article ID e83922, 2013.

[35] O. Shunsuke and T. Hiroshi, "Herbal medicines for the treatment of cancer chemotherapy-induced side effects," Frontiers in Pharmacology, vol. 6, p. 14, 2015.

[36] Y. Xinyu, N. Liu, X. Li et al., "A review on the effect of traditional Chinese medicine against anthracycline-induced cardiac toxicity," Frontiers in Pharmacology, vol. 9, p. 444, 2018.

[37] R. Caballero, R. G. Utrilla, I. Amorós et al., "Tbx20 controls the expression of the KCNH2 gene and of hERG channels," Proceedings of the National Academy of Sciences, vol. 114, no. 3, pp. E416-E425, 2017.

[38] M. Santoni, G. Occhipinti, E. Romagnoli et al., "Different cardiotoxicity of palbociclib and ribociclib in breast cancer: gene expression and pharmacological data analyses, biological basis, and therapeutic implications," BioDrugs: Clinical Immunotherapeutics, Biopharmaceuticals and Gene Therapy, vol. 33, no. 6, pp. 613-620, 2019.

[39] M. A. Tamer, A. Yen-Sin, E. Radzinsky et al., "Regulation of cell cycle to stimulate adult cardiomyocyte proliferation and cardiac regeneration," Cell, vol. 173, no. 1, pp. 104-116, 2018.

[40] C. Brandon, A. Roczkowsky, C. J. Woo et al., "MMP inhibitors attenuate doxorubicin cardiotoxicity by preventing intracellular and extracellular matrix remodeling," Cardiovascular Research, 2020.
[41] C.-Y. Hang, J. Y. Chen, C. H. Kuo et al., "Mitochondrial ROSinduced ERK1/2 activation and HSF2-mediated AT R upregulation are required for doxorubicin-induced cardiotoxicity," Journal of Cellular Physiology, vol. 233, no. 1, pp. 463-475, 2018.

[42] L. Yang, C. Luo, C. Chen, X. Wang, W. Shi, and J. Liu, "Alltrans retinoic acid protects against doxorubicin-induced cardiotoxicity by activating the ERK2 signalling pathway," British Journal of Pharmacology, vol. 173, no. 2, pp. 357-371, 2016.

[43] S. Pandey, J. Bourn, and M. Cekanova, "Mutations of p53 decrease sensitivity to the anthracycline treatments in bladder cancer cells," Oncotarget, vol. 9, no. 47, pp. 28514-28531, 2018.

[44] A. N Bullock and A. R. Fersht, "Rescuing the function of mutant p53," Nature Reviews Cancer, vol. 1, no. 1, pp. 68-76, 2001.

[45] G. Takemura and H. Fujiwara, "Doxorubicin-induced cardiomyopathy," Progress in Cardiovascular Diseases, vol. 49, no. 5, pp. 330-352, 2007.

[46] D. Cappetta, A. De Angelis, L. Sapio et al., "Oxidative stress and cellular response to doxorubicin: a common factor in the complex milieu of anthracycline cardiotoxicity," Oxidative Medicine and Cellular Longevity, vol. 2017, Article ID 1521020, 13 pages, 2017.

[47] M. S. Bin Sayeed and S. S. Ameen, "Beta-sitosterol: a promising but orphan nutraceutical to fight against cancer," Nutrition and Cancer, vol. 67, no. 8, pp. 1216-1222, 2015.

[48] A. A. Basker, A. N. S. Khalid, G. M. Paulraj, A. M. Alsaif, M. A. Muamar, and S. Ignacimuthu, " $\beta$-sitosterol prevents lipid peroxidation and improves antioxidant status and histoarchitecture in rats with 1,2-dimethylhydrazine-induced colon cancer," Journal of Medicinal Food, vol. 15, no. 4, pp. 335-343, 2012.

[49] C.-E. Chen, C.-M. Hsieh, S.-C. Huang, C.-Y. Su, M.-T. Sheu, and H.-O. Ho, "Lecithin-stabilized polymeric micelles $\left(\mathrm{L}_{\mathrm{sb}} \mathrm{PMs}\right)$ for delivering quercetin: pharmacokinetic studies and therapeutic effects of quercetin alone and in combination with doxorubicin," Scientific Reports, vol. 8, no. 1, p. 17640, 2018.

[50] W. Wu, B. Yang, Y. Qiao, Z. Qing, H. He, and M. He, "Kaempferol protects mitochondria and alleviates damages against endotheliotoxicity induced by doxorubicin," Biomedicine \& Pharmacotherapy, vol. 126, p. 110040, 2020.

[51] K. Suchal, S. Malik, N. Gamad et al., "Kampeferol protects against oxidative stress and apoptotic damage in experimental model of isoproterenol-induced cardiac toxicity in rats," Phytomedicine: International Journal of Phytotherapy and Phytopharmacology, vol. 23, no. 12, pp. 1401-1408, 2016.

[52] J. Samanta, A. Mondal, S. Saha, S. Chakraborty, and A. Sengupta, "Oleic acid protects from arsenic-induced cardiac hypertrophy via AMPK/FoxO/NFATc3 pathway," Cardiovascular Toxicology, vol. 20, no. 3, pp. 261-280, 2020.

[53] P. Pittayapruek, J. Meephansan, O. Prapapan, M. Komine, and M. Ohtsuki, "Role of matrix metalloproteinases in photoaging and photocarcinogenesis," International Journal of Molecular Sciences, vol. 17, no. 6, 2016.

[54] Q. Chen, M. Jin, F. Yang, J. Zhu, Q. Xiao, and L. Zhang, "Matrix metalloproteinases: inflammatory regulators of cell behaviors in vascular formation and remodeling," Mediators of Inflammatory, vol. 2013, Article ID 928315, 14 pages, 2013.

[55] Y. Wang, Q. Wang, L. Zhang et al., "Coptisine protects cardiomyocyte against hypoxia/reoxygenation-induced damage via inhibition of autophagy," Biochemical and 
Biophysical Research Communications, vol. 490, no. 2, pp. 231-238, 2017.

[56] L.-L. Gong, L.-H. Fang, S.-B. Wang et al., "Coptisine exert cardioprotective effect through anti-oxidative and inhibition of RhoA/Rho kinase pathway on isoproterenol-induced myocardial infarction in rats," Atherosclerosis, vol. 222, no. 1, pp. 50-58, 2012.

[57] L. Zhou, F. Yang, G. Li et al., "Coptisine induces apoptosis in human hepatoma cells through activating $67-\mathrm{kDa}$ laminin receptor/cGMP signaling," Frontiers in Pharmacology, vol. 9, p. 517, 2018.

[58] W. Zhang, P. Cheng, W. Hu et al., "Inhibition of microRNA384-5p alleviates osteoarthritis through its effects on inhibiting apoptosis of cartilage cells via the NF- $\kappa \mathrm{B}$ signaling pathway by targeting SOX9," Cancer Gene Therapy, vol. 25, no. 11-12, pp. 326-338, 2018.

[59] X. Dong, J. Fu, X. Yin et al., "Emodin: a review of its pharmacology, toxicity and pharmacokinetics," Phytotherapy Research, vol. 30, no. 8, pp. 1207-1218, 2016.

[60] N. Aziz, M.-Y. Kim, and J. Y. Cho, "Anti-inflammatory effects of luteolin: a review of in vitro, in vivo, and in silico studies," Journal of Ethnopharmacology, vol. 225, pp. 342-358, 2018.

[61] C. Yi, G. Li, D. N. Ivanov et al., "Luteolin inhibits Musashi1 binding to RNA and disrupts cancer phenotypes in glioblastoma cells," RNA Biology, vol. 15, no. 11, pp. 1420-1432, 2018.

[62] B. Tian, M. Tian, and S.-M. Huang, "Advances in phytochemical and modern pharmacological research of Rhizoma corydalis," Pharmaceutical Biology, vol. 58, no. 1, pp. 265-275, 2020.

[63] B. Tian, M. Tian, and S.-M. Huang, "Advances in phytochemical and modern pharmacological research of Rhizoma corydalisa," Pharmaceutical Biology, vol. 58, no. 1, pp. $265-275,2020$.

[64] H. Ling, L. Wu, and L. Li, "Corydalis yanhusuo rhizoma extract reduces infarct size and improves heart function during myocardial ischemia/reperfusion by inhibiting apoptosis in rats," Phytotherapy Research: PTR, vol. 20, no. 6, pp. 448-453, 2006.

[65] J. S. Shi, J. X. Yu, X. P. Chen, and R. X. Xu, "Pharmacological actions of gouteng alkaloids, rhynchophylline and isorhynchophylline," ACTA Pharmacologica Sinica, vol. 24, no. 2, pp. 97-101, 2003.

[66] Y. Zhang, Y. Cui, S. Deng et al., "Isorhynchophylline enhances $\mathrm{Nrf}_{2}$ and inhibits MAPK pathway in cardiac hypertrophy," Naunyn-Schmiedeberg's Archives of Pharmacology, vol. 393, no. 2, pp. 203-212, 2020.

[67] Y. Sun, G. B. Lenon, and A. W. H. Yang, "Phellodendri cortex: a phytochemical, pharmacological, and pharmacokinetic review," Evidence-Based Complementary and Alternative Medicine, vol. 2019, Article ID 7621929, 45 pages, 2019.

[68] C. Yang, L. Wenqing, P. Guangchun, J. Yuanying, and C. Yongbing, "Advances in studies on pharmacological actions of proberberine," Drugs \& Clinic, vol. 28, no. 6, pp. 1012-1017, 2013.

[69] L. Wang, W. Zhang, Z. Lu et al., "Functional gene modulebased identification of phillyrin as an anticardiac fibrosis agent," Frontiers in Pharmacology, vol. 11, p. 1077, 2020.

[70] C. Huang, Y. Lin, H. Su, and D. Ye, "Forsythiaside protects against hydrogen peroxide-induced oxidative stress and apoptosis in PC12 cell," Neurochemical Research, vol. 40, no. 1, pp. 27-35, 2015.

[71] L. Tian, C. P. Su, Q. Wang et al., "Chlorogenic acid: a potent molecule that protects cardiomyocytes from TNF- $\alpha$-induced injury via inhibiting NF- $\kappa \mathrm{B}$ and JNK signals," Journal of Cellular and Molecular Medicine, vol. 23, no. 7, pp. 46664678, 2019.

[72] Y. Li, D. Shen, X. Tang et al., "Chlorogenic acid prevents isoproterenol-induced hypertrophy in neonatal rat myocytes," Toxicology Letters, vol. 226, no. 3, pp. 257-263, 2014.

[73] D. Montaigne, X. Marechal, R. Baccouch et al., "Stabilization of mitochondrial membrane potential prevents doxorubicininduced cardiotoxicity in isolated rat heart," Toxicology and Applied Pharmacology, vol. 244, no. 3, pp. 300-307, 2010.

[74] V. Battaglia, A. M. Brunati, C. Fiore et al., "Glycyrrhetinic acid as inhibitor or amplifier of permeability transition in rat heart mitochondria," Biochimica et Biophysica Acta (BBA)-Biomembranes, vol. 1778, no. 1, pp. 313-323, 2008.

[75] S. Upadhyay, A. K. Mantha, and M. Dhiman, "Glycyrrhiza glabra (Licorice) root extract attenuates doxorubicin-induced cardiotoxicity via alleviating oxidative stress and stabilising the cardiac health in H9c2 cardiomyocytes," Journal of Ethnopharmacology, vol. 258, Article ID 112690, 2020. 